\title{
PRIMITIVE HOMOMORPHIC IMAGES OF SEMIGROUPS
}

\author{
T. E. HALL
}

(Received 12 December 1966)

\section{Introduction and summary}

Consideration of the primitive homomorphic images of semigroups arises naturally from their matrix representation theory. (See, for example, papers [1] and [2].) This is basically because a semigroup of matrices, in which all the matrices have the same rank, is necessarily primitive. (The set of idempotents in the semigroup may be empty, however).

In his paper [2], Preston has shown (lemma 3) that a necessary condition for a regular semigroup to have a 0 -restricted primitive homomorphic image is that the semigroup be categorical at zero, and (theorem 5) that for an inverse semigroup, this condition is also sufficient. (The terms "primitive", "categorical at zero", and "0-restricted" are defined below.) Furthermore, for an inverse semigroup $S=S^{0}$, the finest congruence giving a 0 -restricted primitive homomorphic image of $S$ may be written as

$$
\begin{array}{r}
\pi=\{(x, y) \in S \times S: a x=a y \neq 0, x b=y b \neq 0 \\
\text { for some } a, b \in S\} \cup\{(0,0)\} .
\end{array}
$$

(See [2], theorem 5, and the remark at the end of the proof.)

In this paper, we show, by a direct method, that if a semigroup $S=S^{0}$ is categorical at zero, then it has a maximal 0 -restricted primitive homomorphic image; if $S$ is also regular, then this image is $S / \pi^{*}$, where $\pi^{*}$ is the congruence on $S$ generated by the relation $\pi$ above.

\section{Semigroups which are categorical at zero}

We repeat firstly some definitions from [2]. A semigroup $S=S^{0}$ is primitive if each of its non-zero idempotents is primitive. A semigroup $S=S^{0}$ is categorical at zero if $a b c=0$ implies either $a b=0$ or $b c=0$, for any elements $a, b, c$ of $S$. A congruence $\rho$ on a semigroup $S=S^{0}$ is 0 -restricted if $\{0\}$ is a $\rho$-class. A homomorphism $\phi$ of $S=S^{0}$ is 0 -restricted if the congruence $\phi \circ \phi^{-1}$ naturally induced on $S$ by $\phi$ is 0 -restricted. 
Let $S=S^{0}$ be a semigroup which is categorical at zero.

Define

$$
\begin{aligned}
& \rho_{1}=\left\{(e, f) \in S \times S: e^{2}=e, f^{2}=f, e f=f e=f \neq 0\right\}, \\
& \rho_{1}^{\prime}=\rho_{1} \cup \rho_{1}^{-1} \cup \iota_{S},
\end{aligned}
$$

where $\iota_{S}$ is the identity relation on $S$,

$$
\begin{aligned}
& \rho_{1, c}=\{(x, y) \in S \times S: x=u a v, y=u b v, \\
& \text { for some } \left.(a, b) \in \rho_{1}^{\prime}, \text { and for some } u, v \in S^{1} .\right\}
\end{aligned}
$$

and

$$
\rho_{1}^{*}=\rho_{1, c}^{t}=\rho_{1, c} \cup\left(\rho_{1, c} \circ \rho_{1, c}\right) \cup\left(\rho_{1, c} \circ \rho_{1, c} \circ \rho_{1, c}\right) \cup \cdots .
$$

$\rho_{1}^{*}$ is the congruence on $S$ generated by the relation $\rho_{1} \cdot([3]$, p. 18). We show now that $\rho_{1}^{*}$ is 0 -restricted. We show first that

(i) if $(e, f) \in \rho_{1}$, and $u, v \in S$, then $u e v=0$ if and only if $u f v=0$.

Suppose $u e v=0$. Then since $S$ is categorical at zero,

which implies

$$
u e=0 \text { or } e v=0 \text {, }
$$

i.e.

$$
u f=u e f=0 \quad \text { or } \quad f v=f e v=0,
$$

Suppose $u f v=0$. Then

$$
u f v=0 .
$$

i.e.

$$
u f=0 \text { or } \quad f v=0,
$$

which means

$$
u e f=0 \text { or } f e v=0,
$$

since $e f=f e=f \neq 0$ i.e.

$$
u e=0 \text { or } e v=0
$$

Setting $v=e$ we obtain

$$
u e v=0 .
$$

$$
u e=0 \text { if and only if } u f=0 \text {, for any }(e, f) \in \rho_{1}, u \in S,
$$

and setting $u=e$ we obtain

(iii) $\quad e v=0$ if and only if $f v=0$, for any $(e, f) \in \rho_{1}, v \in S$.

From (i), (ii) and (iii) we easily see that $u a v=0$ if and only if $u b v=0$, for any $(a, b) \in \rho_{1}^{\prime}, u, v \in S^{1}$. This means that $(0,0)$ is the only pair in $\rho_{1,0}$ which contains 0 , and this then is also true of $\rho_{1}^{*}$, i.e., $\rho_{1}^{*}$ is 0 -restricted. $S / \rho_{1}^{*}$ is thus also categorical at zero, but is not necessarily primitive.

Define now by induction $\rho_{m+1}^{*}$, the congruence generated by

$$
\rho_{m+1}=\left\{(e, f) \in S \times S: e^{2} \rho_{m}^{*} e, f^{2} \rho_{m}^{*} f \text {, ef } \rho_{m}^{*} f e \rho_{m}^{*} f \neq 0\right\} .
$$


Then by using the method above, and considering the images of $e, f, u, v$ in $S / \rho_{m}^{*}$, where $(e, f) \in \rho_{m+1}, u, v \in S$, we can easily show that $\rho_{m+1}^{*}$ is 0 restricted, if we assume $\rho_{m}^{*}$ to be 0 -restricted. By induction we have that $\rho_{n}^{*}$ is 0 -restricted for all natural numbers $n$. Define now

$$
\rho=\bigcup_{n=1}^{\infty} \rho_{n}^{*}
$$

Clearly $\rho$ is 0 -restricted and, since $\rho_{n}^{*} \subseteq \rho_{n+1}^{*}$ for each $n$, is also a congruence. It is an easy matter to show that $S / \rho$ is primitive; for let us consider any idempotents $E, F \in S / \rho$ such that $E F=F E=F \neq 0$. Choose any elements $e \in E, f \in F$. Then $e \rho e^{2}, f \rho f^{2}$, and ef $\rho f e \rho f \neq 0$. Since $\rho=\bigcup_{n=1}^{\infty} \rho_{n}^{*}$, there exist numbers $n_{1}, n_{2}, n_{3}, n_{4}$, such that $e \rho_{n_{1}}^{*} e^{2}, f \rho_{n_{2}}^{*} f^{2}$, ef $\rho_{n_{3}}^{*}$ fe and $f e \rho_{n_{4}}^{*} f$. Choosing $i=\max \left\{n_{1}, n_{2}, n_{3}, n_{4}\right\}$, and using $\rho_{n}^{*} \subseteq \rho_{n+1}^{*}$, we obtain $e \rho_{i}^{*} e^{2}$, $f \rho_{i}^{*} f^{2}$, ef $\rho_{i}^{*}$ fe $\rho_{i}^{*} f \neq 0$, whence $(e, f) \in \rho_{i+1} \subseteq \rho$, i.e. $E=F$, and so $S / \rho$ is primitive.

If $\beta$ is a 0 -restricted primitive congruence on $S$, it must contain $\rho_{1}^{*}$, and if it contains $\rho_{m}^{*}$, it must contain $\rho_{m+1}^{*}$, and so by induction it contains $\rho$. We have thus proved the following result.

THEOREM 1. Let $S=S^{0}$ be a semigroup, categorical at zero. Then the relation $\rho$, defined by the equations (1), (2) and (3), is the finest 0-restricted primitive congruence on $S$.

\section{Semigroups which are regular and categorical at zero}

Let $S=S^{0}$ be a semigroup which is regular and categorical at zero. Define on $S$ the congruences $\rho_{1}^{*}$ and $\rho$ as before. Also define

$$
\pi=\{(x, y) \in S \times S: a x=a y \neq 0, x b=y b \neq 0, \text { for some } a, b \in S\}
$$

and denote by $\pi^{*}$ the congruence on $S$ generated by $\pi$.

THEOREM 2. Let $S=S^{0}$ be a regular semigroup, categorical at zero. Then $\rho=\rho_{1}^{*}=\pi^{*}$, as defined by equations (1), (2), (3) and (4); whence each is the finest 0-restricted primitive congruence on $S$.

Proof. We show first that $\rho=\rho_{1}^{*}$. For this it will suffice to show that $S / \rho_{1}^{*}$ is primitive. Take any elements $E, F$ of $S / \rho_{1}^{*}$ such that $E^{2}=E$, $F^{2}=F$ and $E F=F E=F \neq 0$. Since $E^{2}=E, F^{2}=F$, from Lallement's result, lemma 2.2, paper [4], there exist idempotents $e, f \in S$, such that $e \in E, f \in F$. We thus have $e^{2}=e, f^{2}=f$, and $e f \rho_{1}^{*} f e \rho_{1}^{*} f \neq 0$. Let $(e f)^{\prime}$ be an inverse of $e f$. Then $e f(e f)^{\prime} e f=e f \neq 0$ and so $e f(e f)^{\prime} e \neq 0$. Now

and

$$
\left[e f(e f)^{\prime} e\right]\left[e f(e f)^{\prime} e\right]=e f(e f)^{\prime} e
$$


Thus

$$
e\left[e f(e f)^{\prime} e\right]=\left[e f(e f)^{\prime} e\right] e=\left[e f(e f)^{\prime} e\right] \neq 0 .
$$

whence

$$
e \rho_{1}^{*} e f(e f)^{\prime} e \text {, }
$$

$$
f e \rho_{1}^{*} f e f(e f)^{\prime} e \rho_{1}^{*} f(e f)^{\prime} e \rho_{1}^{*} e f(e f)^{\prime} e \rho_{1}^{*} e .
$$

But $f e \rho_{1}^{*} f$, so $f \rho_{1}^{*} e$, i.e. $E=F$, and $S / \rho_{1}^{*}$ is primitive, and so we have

$$
\rho=\rho_{1}^{*} \text {. }
$$

Now choose any pair $(x, y) \in \pi$. Then there exist elements $a, b \in S$ such that $a x=a y \neq 0$, and $x b=y b \neq 0$. Also there exist elements $A, B, X, Y$ in $S / \rho_{1}^{*}$ such that $a \in A, b \in B, x \in X, y \in Y$, and then $A X=A Y \neq 0$ and $X B=Y B \neq 0$ since $\rho_{1}^{*}$ is 0 -restricted. Since $S / \rho_{1}^{*}$ is primitive and regular, it is the 0 -direct union of a set of completely 0 -simple subsemigroups, from Preston's paper [2], theorem 1. Clearly $A, B, X, Y$ are in the same completely 0 -simple subsemigroup, and this means that $X=Y$, i.e.

Therefore

$$
(x, y) \in \rho_{1}^{*} \text {. }
$$

whence

$$
\begin{gathered}
\pi \subseteq \rho_{1}^{*}, \\
\pi^{*} \subseteq \rho_{1}^{*} .
\end{gathered}
$$

But clearly $\rho_{1} \subseteq \pi$, whence $\rho_{1}^{*} \subseteq \pi^{*}$. Therefore

$$
\pi^{*}=\rho_{1}^{*} \text {. }
$$

This completes the proof of the theorem.

By a method similar to that used above, it can also be shown that for periodic semigroups which are categorical at zero,

$$
\rho=\rho_{1}^{*} \text {. }
$$

\section{Acknowledgement}

I wish to thank my supervisor Professor G. B. Preston for pointing out these problems to me and for his many valuable suggestions.

Note added in proof. Since this paper was submitted, the paper 'Matrix representations of semigroups' by D. B. McAlister (Glasgow Math. $J .8(1967), 1-13)$, has appeared. It contains strongly related results.

\section{References}

[1] W. D. Munn, Matrix representations of inverse semigroups, Proc. London Math. Soc. 14 (1964), 165-181.

[2] G. B. Preston, Matrix representations of inverse semigroups, J. Aust. Math. Soc. (to appear). 
[3] A. H. Clifford and G. B. Preston, The Algebraic Theory of Semigroups (Math. Surveys, number 7, Amer. Math. Soc. 1961).

[4] G. Lallement, Congruences et équivalences de Green sur un demi-groupe régulier, C. R. Acad. Sc. Paris, Série A, 262 (1966), 613-616.

Department of Mathematics

Monash University, Clayton 